\title{
Role of imaging in predicting tumor spread through airspaces (STAS): what are the next steps
}

\author{
Constance de Margerie-Mellon ${ }^{1}$, Paul A. VanderLaan ${ }^{2}$, Benedikt H. Heidinger ${ }^{1,3}$, Alexander A. Bankier ${ }^{1}$ \\ ${ }^{1}$ Department of Radiology, Beth Israel Deaconess Medical Center and Harvard Medical School, Boston, MA, USA; ${ }^{2}$ Department of Pathology, Beth \\ Israel Deaconess Medical Center and Harvard Medical School, Boston, MA, USA; ${ }^{3}$ Department of Biomedical Imaging and Image-guided Therapy, \\ Medical University of Vienna, Vienna, Austria \\ Correspondence to: Constance de Margerie-Mellon, MD. Beth Israel Deaconess Medical Center, 330 Brookline Ave., Boston, MA 02215, USA. \\ Email: constancedemm@gmail.com. \\ Provenance and Peer Review: This is an invited article commissioned and reviewed by the Section Editor Jun Zhou (Department of Nuclear Medicine, \\ Zhongshan Hospital, Fudan University, Shanghai, China). \\ Response to: Johnston RP, Emoto K, Dux J, et al. Predicting spread through air spaces (STAS) preoperatively: can imaging help? J Thorac Dis \\ 2019;11:S1938-41.
}

Submitted Oct 25, 2019. Accepted for publication Nov 26, 2019.

doi: $10.21037 /$ jtd.2019.11.20

View this article at: http://dx.doi.org/10.21037/jtd.2019.11.20

Tumor spread through airspaces (STAS) has been identified as a new pattern of invasion in the 2015 WHO classification of lung tumors (1) and is associated with a lower overall survival in resected non-small cell lung cancers (2). As a consequence, sublobar resection may not be the best surgical option for cancers with STAS. Preoperatively identifying STAS in resectable lung cancers would, therefore, be of clinical relevance. In their recent Editorial, Johnston et al. summarized the findings of several retrospective radiological-pathological correlation studies on this topic (3). Overall, imaging features associated with STAS include pure solid or predominantly solid CT patterns of the cancers, as well as a bigger size and a higher $\mathrm{SUV}_{\max }$ on PET-CT (4-8). However, there are also discrepancies between studies as to the imaging findings related to STAS. These discrepancies touch on the likelihood of STAS in cancers presenting as pure ground glass nodules or as partsolid nodules with small solid components (4-8). Although the publications analyzed in the above editorial are certainly thought-provoking and in part encouraging, they also highlight that the role of CT in the preoperative assessment of STAS is not clear yet. What are potential steps required to more firmly establish CT in the preoperative diagnosis of STAS?

A first step would certainly be a more stringent pathological definition of STAS. There are, in fact, differences in how the authors of the studies discussed in the Editorial define STAS. Some authors define STAS as the presence of tumor cells beyond the edge of the main tumor $(4,6)$. Other authors choose a more detailed definition for STAS, by incorporating a minimum distance of at least $0.5 \mathrm{~cm}$ between the tumor edge and the tumor cells (7), or by excluding single remote tumor cells or clusters from the definition (5). As a consequence, tumor cells located $0.4 \mathrm{~cm}$ away from the main tumor edge could be considered as STAS according to some authors $(4,6)$, but not according to others (7). The incidence of STAS in lung adenocarcinoma reported in the literature varies greatly, from $12 \%$ to $100 \%(7,9)$. This variation may be the result of different populations, but it also may be the result of varying definitions for STAS. Likewise, it is conceivable that variations in the definition of STAS could contribute to discrepancies between studies in STAS-associated CT features. Generally agreeing on a comprehensive definition for STAS would help establish a stronger reference for pathological-radiological correlation studies.

Second, in pathological-radiological correlation studies, it may be worth looking beyond the relatively basic CT morphological parameters investigated so far. To date, these basic morphological CT parameters mainly rely on 2-dimensional size measurements or on relatively straightforward qualitative CT features such as solid, part- 
solid, or non-solid CT morphology. STAS is an oftentimes subtle histological feature, well below the spatial resolution of current imaging techniques. It therefore is unlikely that current imaging approaches with less spatial resolution will be able to grasp or even mirror the full complexity of STAS. The associations between larger nodule size or higher percentage of solid component, for instance, and the presence of STAS are certainly convincing. However, these associations alone do not justify conceptualizing CT morphological parameters as clinical biomarkers of STAS. As suggested by Johnston et al., more advanced, quantitative imaging parameters may be needed to better characterize signs suggestive of STAS on CT. These parameters could focus on the 3-dimensional peritumoral area, where the STAS phenomenon occurs on a histological level. Histogram-based analysis could provide data about the peripheral attenuation of the tumor, whereas the heterogeneity of the peri-tumoral area could reflect in texture-derived parameters. Such parameters could potentially be used by researchers together with clinical and morphological CT parameters, in order to build stronger predictive models for STAS.

Finally, looking for an alternate path to the pre-surgery identification of STAS, the authors mention the added value of intraoperative frozen sections. They cite a study from their team in which they obtained a $71 \%$ sensitivity and a $92 \%$ specificity for the identification of STAS on frozen sections (10). These are encouraging results. However, another team published less optimistic results, with a sensitivity of $50 \%$ and a negative predictive value of $8 \%$, largely insufficient to rule out STAS during surgery (9). These discrepancies underline the challenge in identifying STAS on frozen sections. Frozen section performances have not yet been compared to CT performances for the identification of parameters associated with STAS. Taking into account CT morphological and/or quantitative parameters associated with STAS when assessing frozen sections could help the pathologist identify cases where more extensive frozen section sampling may be warranted to identify STAS. Those parameters could also help to decrease diagnostic uncertainty in the pre-surgical diagnosis of STAS and should be tested in future studies.

Discussion about STAS inserts into the continuous refinement of the concept of invasiveness, both in pathology and radiology. This could explain the surge in the interest in STAS, but also in other aspects of invasiveness, for example in pleural invasion (11). This also parallels the increasing morphological complexity of cancers, the components of which are studied with increasing sophistication on imaging. Both, questions and answers, about the relation between imaging findings and STAS ultimately emphasize that our next steps will require an even closer collaboration between the imaging and the pathology community.

\section{Acknowledgments}

Funding: None.

\section{Footnote}

Conflicts of Interest: C. de Margerie-Mellon received grants from Institut Servier and Olea Medical; P. VanderLaan has received consulting fees from Gala Therapeutics and from Foundation Medicine; A. Bankier is a consultant to Daiichi Sankyo Company, Hummingbird Diagnostics and Olympus, all outside the submitted work. B. Heidinger has no conflicts of interest to declare.

Ethical Statement: The authors are accountable for all aspects of the work in ensuring that questions related to the accuracy or integrity of any part of the work are appropriately investigated and resolved.

Open Access Statement: This is an Open Access article distributed in accordance with the Creative Commons Attribution-NonCommercial-NoDerivs 4.0 International License (CC BY-NC-ND 4.0), which permits the noncommercial replication and distribution of the article with the strict proviso that no changes or edits are made and the original work is properly cited (including links to both the formal publication through the relevant DOI and the license). See: https://creativecommons.org/licenses/by-nc-nd/4.0/.

\section{References}

1. Travis WD, Brambilla E, Nicholson AG, et al. The 2015 World Health Organization Classification of Lung Tumors Impact of Genetic, Clinical and Radiologic Advances Since the 2004 Classification. J Thorac Oncol 2015;10:1243-60.

2. Chen D, Mao Y, Wen J, et al. Tumor Spread Through Air Spaces in Non-Small Cell Lung Cancer: a systematic review and meta-analysis. Ann Thorac Surg 2019;108:945-54.

3. Johnston RP, Emoto K, Dux J, et al. Predicting spread through air spaces (STAS) preoperatively: can imaging help? J Thorac Dis 2019;11:S1938-41. 
4. de Margerie-Mellon C, Onken A, Heidinger BH, et al. CT Manifestations of Tumor Spread Through Airspaces in Pulmonary Adenocarcinomas Presenting as Subsolid Nodules. J Thorac Imaging 2018;33:402-8.

5. Toyokawa G, Yamada Y, Tagawa T, et al. Computed tomography features of resected lung adenocarcinomas with spread through air spaces. J Thorac Cardiovasc Surg 2018;156:1670-6.e4.

6. Kim SK, Kim TJ, Chung MJ, et al. Lung Adenocarcinoma: CT Features Associated with Spread through Air Spaces. Radiology 2018;289:831-40.

7. Shiono S, Yanagawa N. Spread through air spaces is a predictive factor of recurrence and a prognostic factor in stage I lung adenocarcinoma. Interact Cardiovasc Thorac Surg 2016;23:567-72.

8. Kameda K, Lu S, Eguchi T, et al. MA12.05 Can Tumor Spread through Air Spaces (STAS) in Lung

Cite this article as: de Margerie-Mellon C, VanderLaan PA, Heidinger BH, Bankier AA. Role of imaging in predicting tumor spread through airspaces (STAS): what are the next steps. J Thorac Dis 2020;12(3):1154-1156. doi: 10.21037/jtd.2019.11.20
Adenocarcinomas Be Predicted Pre- and Intraoperatively? J Thorac Oncol 2017;12:S411-2.

9. Walts AE, Marchevsky AM. Current Evidence Does Not Warrant Frozen Section Evaluation for the Presence of Tumor Spread Through Alveolar Spaces. Arch Pathol Lab Med 2018;142:59-63.

10. Eguchi T, Kameda K, Lu S, et al. Lobectomy Is Associated with Better Outcomes than Sublobar Resection in Spread through Air Spaces (STAS)-Positive T1 Lung Adenocarcinoma: A Propensity Score-Matched Analysis. J Thorac Oncol 2019;14:87-98.

11. Heidinger BH, Schwarz-Nemec U, Anderson KR, et al. Visceral Pleural Invasion in Pulmonary Adenocarcinoma: Differences in CT Patterns between Solid and Subsolid Cancers. Radiol Cardiothorac Imaging 2019;1. doi: 10.1148/ryct.2019190071. 